\title{
Coupling Substrate-Integrated Waveguides to Increase the Gain Bandwidth of Leaky-Wave Antennas
}

\author{
Miguel Poveda-García, Student Member IEEE, David Cañete-Rebenaque, Member IEEE, George \\ Goussetis, Senior Member IEEE, José Luis Gómez-Tornero, Senior Member IEEE.
}

\begin{abstract}
A novel technique to increase the pattern bandwidth of substrate integrated waveguide leaky-wave antennas (SIW LWAs) is proposed. By coupling several SIWs, it is shown that the gain at the desired angle can be kept stable across a wide frequency band. A systematic design methodology based on a simple transverse equivalent network is presented. Practical coupledSIW designs with gain exceeding $10 \mathrm{~dB}$ and pointing at $30^{\circ}$ in the $15 \mathrm{GHz}$ band, are reported to validate the theory. Simulated and experimental results demonstrate an enhancement of the halfpower gain bandwidth from $2.5 \%(380 \mathrm{MHz})$ in the single-SIW design to $9 \%(1360 \mathrm{MHz})$ for a LWA composed of three coupled SIWs.
\end{abstract}

Index Terms - Broadband antennas, frequency beam squint, leaky-wave antennas, substrate integrated waveguide.

\section{INTRODUCTION}

$\mathrm{F}$ REQUENCY-SQUINT is a well-known property of leaky-wave antennas (LWAs), due to their characteristic frequency scanning behavior [1]. It might be a useful characteristic for particular scenarios such as frequency modulated-continuous wave (FMCW) radars [2]-[4], or frequency-steered near-field focusing systems [5]. However, the beam squint is an undesired feature which limits the practical bandwidth in important applications such as highly-directive point-to-point telecommunication radio links. For this reason, many efforts have recently been placed to design high-gain scanning LWAs with reduced frequency-scanning sensitivity [6]-[15]. A. Neto [6],[7] proposed a nondispersive printed leaky slot line embedded in a circularly symmetric elliptical dielectric lens which focus the radiated fields to a constant beam direction over a wide frequency range. However, this solution lacks the simple two-dimensional structure of planar LWAs. Planar LWAs using metamaterial unit-cells have been proposed to modify the frequency dispersion and reduce the beam squint [8]-[11]. Anisotropic meta-substrates [12] and non-reciprocal ferritebased metamaterials [13] have also proven beam squint reduction. Finally, active circuits can provide non-Foster dispersion responses for squint-free LWAs operating at an off-

Manuscript received October 5, 2017; revised January 14, 2018. This work has been supported by Spanish National project TEC2013-47037-C5-5-R, TEC2016-75934-C4-4-R, and Regional Seneca project 19494/PI/14.

M. Poveda-García, D. Cañete-Rebenaque and J.L. Gómez-Tornero are with the Group of Electromagnetism Applied to Telecommunications (GEAT), broadside angle [14], [15]. In this paper we describe a totally different technique to improve the gain bandwidth of planar LWAs in substrate integrated waveguide (SIW) technology. The proposed technique dispenses the need of bulky dielectric lenses, metamaterial unit-cells, anisotropic materials, or active circuits. The novel structure is based on the SIW LWA [16] shown in Fig. 1a, which is modified by adding extra longitudinally coupled SIWs ([17], [18]) as shown in Fig. 1.

This coupled SIW LWA topology is inspired by a similar multi-layer arrangement used for Fabry-Pérot cavity antennas (FPA) [19]-[25]. The addition of extra coupled FP cavities has demonstrated increased pattern bandwidth for high-gain broadside radiation compared to the original single-cavity FPA. In the same manner, in this paper it is demonstrated for the first time that the high-gain radiation pattern of the single SIW LWA (Fig. 1a), can be improved in terms of gain bandwidth at a given off-broadside elevation angle by increasing the number of coupled SIWs. The design theory is based on a simple Transverse Equivalent Network (TEN) which is optimized to satisfy the associated phase resonance condition for the desired angle over a wide band, as described in Section II. Practical examples of coupled SIW LWAs operating at the frequency of $15 \mathrm{GHz}$ and pointing at a $30^{\circ}$ angle are presented in Section III, illustrating that higher bandwidth with squint-free condition is obtained as the number of coupled SIWs (N) is increased from 1 to 3. Finally, Section IV reports experimental validation performed on manufactured prototypes, and Section V presents the conclusions of this work.

Previous results presented in [17], [18] have been improved with the following contributions. First, a polynomial fitting of the transverse equivalent network components is proposed to reduce the time of optimization. Second, we use the half-power gain bandwidth definition to quantitatively demonstrate the improvement in the antenna bandwidth for the desired scanning angle. Third, detailed description of the optimization process to obtain the highest possible gain bandwidth is explained in section III.B. Finally, experimental demonstration is also reported for the first time in this paper.

Technical University of Cartagena (UPCT), Cartagena 30202, Spain (e-mail: miguel.poveda@upct.es, david.canete@upct.es, josel.gomez@upct.es).

G. Goussetis is with the Institute of Sensors Signals and Systems, HeriotWatt University, Edinburgh EH14 4AS, UK (e-mail: g.goussetis@hw.ac.uk) 

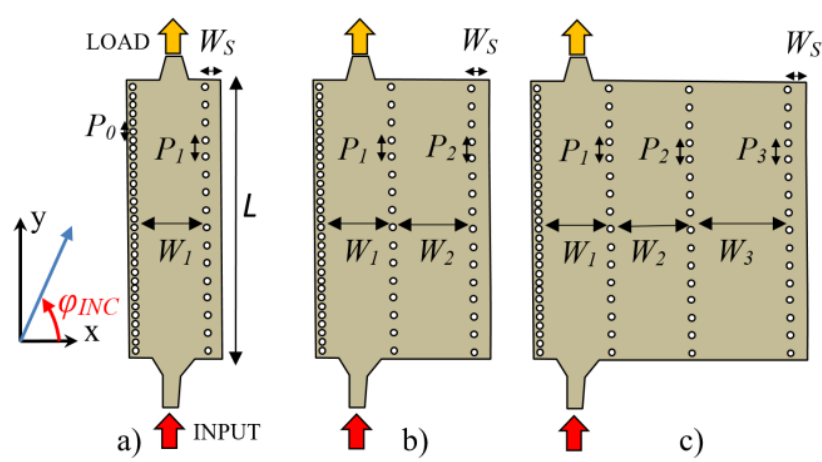

Fig. 1. Scheme of coupled SIW LWA of order a) $\mathrm{N}=1$ b) $\mathrm{N}=2$ c) $\mathrm{N}=3$.

\section{DESIGN THEORY}

The structure of the proposed coupled SIW LWA is illustrated in Fig. 1. The original SIW LWA [16] shown in Fig. $1 \mathrm{a}$ is formed by a single SIW of width $W_{l}$ created between a dense row of vias of diameter $d$ and periodicity $P_{0}$ acting as totally reflective wall, and a partially reflective sheet (PRS) of vias with similar diameter $d$ and located at a bigger distance $P_{I}$ to allow for power leakage. Radiation occurs at the end fringe of the strip of width $W_{S}$ located at the right side of the SIWs in Fig. 1. A quasi-TE 10 leaky mode with dispersive complex longitudinal propagation constant $k_{y}(f)$ can model this continuous radiation along the antenna length:

$$
k_{y}(f)=\beta_{y}(f)-j \alpha_{y}(f)
$$

The antenna length $\mathrm{L}$ behaves as a magnetic-current linesource that radiates in the form of a fan beam. If low-leakage condition is assumed $\left(\beta_{y} \gg \alpha_{y}\right)$, the elevation angle $\theta_{R}$ (measured from broadside, see Fig. 2a) and the $3 \mathrm{~dB}$ beamwidth $\Delta \theta$, are related to the leaky-mode phase constant $\beta_{y}$ by the following equations [1]:

$$
\begin{gathered}
\sin \theta_{R}(f) \approx \frac{\beta_{y}(f)}{k_{0}}=c_{0} \frac{\beta_{y}(f)}{2 \pi f} \\
\Delta \theta_{R}(f) \approx \frac{1}{L / \lambda_{0} \cos \theta_{R}(f)}
\end{gathered}
$$

where $k_{0}$ is the free-space wavenumber, $\lambda_{0}$ is the free-space wavelength, and $c_{0}$ is the speed of light in air. To eliminate the frequency-beam squint, the angle $\theta_{R}$ must be fixed to a constant goal value for all frequencies, so from (2) we obtain a linear dependence with frequency for $\beta_{y}(f)$ that must be satisfied:

$$
\theta_{R}(f)=\theta_{G O A L} \rightarrow \beta_{y}(f)=\beta_{y G O A L}(f)=\frac{2 \pi f}{c_{0}} \sin \theta_{G O A L}
$$

To analyze the leaky-mode phase constant dispersion $\beta_{y}(f)$, a simple Transverse Equivalent Network (TEN) along the $\mathrm{x}$ direction proposed in [26] and shown in Fig. 2 can be used.

A T-junction network formed by two series capacitances $C_{S}$

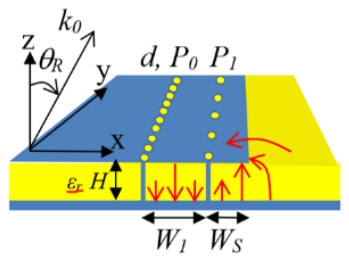

a)

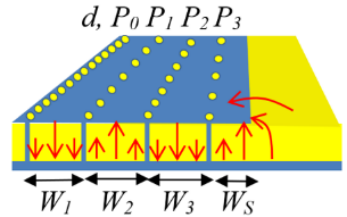

b)

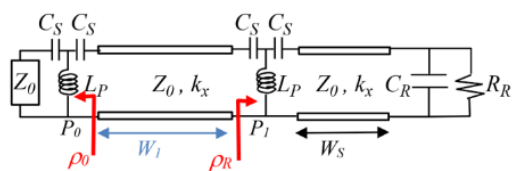

c)

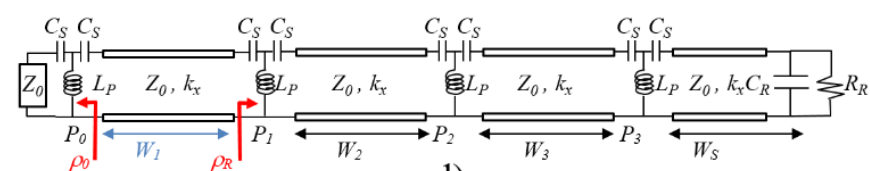

d)

Fig. 2. 3D view of a coupled-SIW LWA of order a) $\mathrm{N}=1$ b) $\mathrm{N}=3$ and associated Transverse Equivalent Network (TEN) c) $\mathrm{N}=1 \mathrm{~d}$ ) $\mathrm{N}=3$. Red lines in a) and b) represent the fundamental TE leaky-mode electric field distribution.

and a parallel inductance $L_{P}$ given by Marcuvitz [27] is used to model each row of conducting vias of diameter $d$ and for any periodicity $P$. The strip fringe radiation impedance is modeled by a shunt capacitor $C_{R}$ and a shunt resistance $R_{R}$, which values are computed for the SIW host substrate of height $H$ and relative dielectric constant $\varepsilon_{r}$ using Kuester approximation for thin substrates [28]. It must be taken into account that the values for $C_{S}, L_{P}, C_{R}$ and $R_{R}$ depend on the internal angle of incidence $\varphi_{I N C}$ shown in Fig. 1a [26], which is related to the leaky radiation angle $\theta_{R}$ and phase constant $\beta_{y}$ by the Snell equation:

$$
\sin \varphi_{I N C}(f)=\frac{\sin \theta_{R}(f)}{\sqrt{\varepsilon_{r}}} \approx \frac{\beta_{y}(f)}{k_{0} \sqrt{\varepsilon_{r}}}
$$

For the efficient design of the multi-cavity SIW LWA, the values of $C_{S}, L_{P}$ are first computed for a range of posts periodicity values $P$ using an accurate in-house tool based on the Method of Moments (MoM) [26], and for any fixed incidence angle $\varphi_{I N C}$. Then a second-degree polynomial fitting is carried out to obtain closed-form expressions for the component values as a function of $P$ :

$$
\begin{gathered}
L_{P}(P)=a_{L} P^{2}+b_{L} P+c_{L} \\
C_{S}(P)=a_{C} P^{2}+b_{C} P+c_{C}
\end{gathered}
$$

Fig. 3 represents these functions for an internal angle of incidence $\varphi_{I N C}=19^{\circ}$, as a function of $P$ in the range from $P=2$ $\mathrm{mm}$ to $P=7 \mathrm{~mm}$, and for a post diameter $d=1 \mathrm{~mm}$. They are compared with MoM results showing good agreement in the full range of values of $P$. Clearly the use of closed-form analytical expressions lowers the computational cost needed compared to full-wave MoM analysis. This time reduction is indispensable for the subsequent optimization technique, which needs hundreds of analyses to properly design the distance $P$ between coupling posts for each row of coupling vias. 


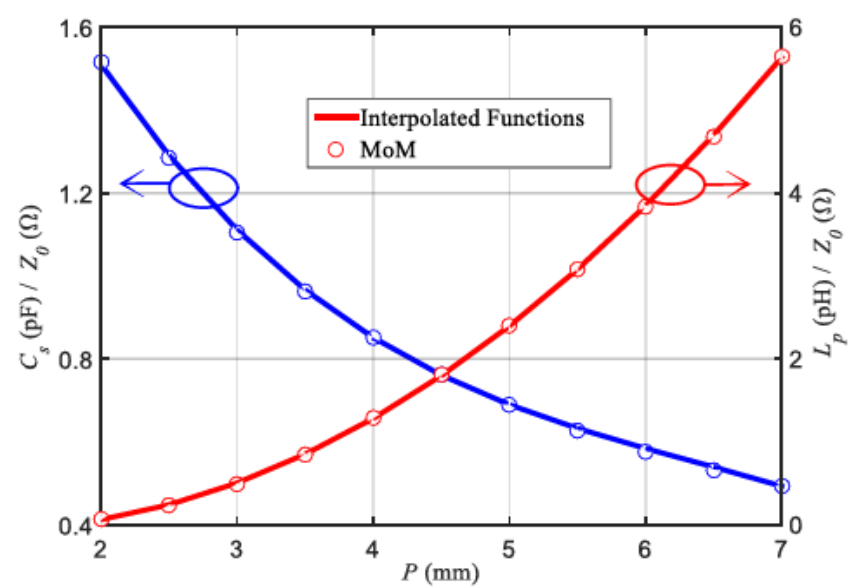

Fig. 3. Variation of the $C_{S}$ and $L_{P}$ as a function of vias periodicity $P$ and frequency for a fixed incidence angle $\varphi_{I N C}=19$, with $d=1 \mathrm{~mm}$.

Next, the SIWs are modelled in the TEN by transmission lines of length Wi, TE characteristic impedance $Z_{0}=1 / \omega k_{x}$ and complex transverse propagation constant $k_{x}(f)$ related to the leaky-mode longitudinal constant $k_{y}(f)(1)$ by:

$$
k_{x}(f)=\sqrt{k_{0}{ }^{2} \varepsilon_{r}-k_{y}(f)^{2}}=\beta_{x}(f)+j \alpha_{x}(f)
$$

Under low-leakage conditions $\left(\beta_{y} \gg \alpha_{y}\right)$ and using (2), (8) can be rewritten as a function of frequency and the angle $\theta_{R}$ :

$$
k_{x}(f) \approx \beta_{x}(f)=\frac{2 \pi f}{c_{0}} \sqrt{\varepsilon_{r}-\sin ^{2} \theta_{R}(f)}
$$

so that the transverse phase constant $\beta_{x}(f)$ must follow the next linear function with frequency to satisfy the squint-free goal condition (4):

$$
\beta_{x G O A L}(f)=\frac{2 \pi f}{c_{0}} \sqrt{\varepsilon_{r}-\sin ^{2} \theta_{G O A L}}
$$

The leaky-mode dispersion is given by the transverse resonance equation (TRE):

$$
\rho_{0} e^{-j 2 k_{x} W_{1}} \rho_{R}=1
$$

where $\rho_{R}$ and $\rho_{0}$ stand for the reflection coefficients at the right and left sides of the SIW of width $W_{l}$ (see Fig. 2). Inserting (10) in (11) and taking phases, the following goal function $\Psi_{\text {GOAL }}(f)$ is obtained for the phase of the reflection coefficient $\rho_{R}$, as a function of frequency and for the goal angle $\theta_{G O A L}$, and a given width $W_{1}$ :

$$
\begin{aligned}
& \Psi_{G O A L}(f)= \\
& =\frac{4 W_{1} \pi f}{c_{0}} \sqrt{\varepsilon_{r}-\sin ^{2} \theta_{G O A L}}-\varphi_{0}\left(f, \varphi_{G O A L}\right)+2 \pi q
\end{aligned}
$$

where $q$ is any integer value, and $\varphi_{0}$ is the phase of $\rho_{0}$ which depends on frequency and on the internal incidence angle $\varphi_{I N C}$.
As for the radiation angle $\theta_{R}$, the internal angle of incidence $\varphi_{I N C}$ is also fixed to a goal value to keep the squint-free condition. Inserting (4) in (5) we obtain:

$$
\varphi_{I N C}(f)=\varphi_{G O A L}=\arcsin \frac{\sin \theta_{G O A L}}{\sqrt{\varepsilon_{r}}}
$$

The design goal is thus to determine the coupled SIWs dimensions (distances between coupling vias $P_{i}$, and SIW widths $W_{i}$ ) which satisfy over a wideband the reflection-phase condition $\Psi_{R}(f)=\Psi_{G O A L}(f)$, or in other words, which minimize the following error function for the reflection phases:

$$
\Delta \Psi\left(f, W_{i}, P_{i}\right)=\Psi_{G O A L}\left(f, W_{1}\right)-\Psi_{R}\left(f, W_{i>1}, P_{i}\right)
$$

It is noted that the first SIW width $W_{l}$ determines the goal reflection phase $\Psi_{G O A L}$, while the rest of SIW widths $W_{i}$ with i $>1$ and the distance between coupling vias $P_{i}$, determine the reflection phase of the right part of the circuit $\Psi_{R}$. The design methodology is described in the next section with practical examples.

\section{DESIGN EXAMPLES}

The SIW substrate is set to $H=0.508 \mathrm{~mm}, \varepsilon_{r}=2.2$, and the diameter of the vias is fixed to $d=1 \mathrm{~mm}$. The row of vias at the left is set to a distance $P_{0}=2 \mathrm{~mm}$ so that leakage is prevented from this side. The desired pointing angle is chosen to $\theta_{G O A L}=30^{\circ}$ and the design frequency is $15 \mathrm{GHz}$. The internal incidence angle (13) is then fixed to $\varphi_{\text {GOAL }}=19^{\circ}$. The following subsections illustrate how an increased number of coupled SIWs (from $\mathrm{N}=1$ to $\mathrm{N}=3$ ) can be optimized to provide higher squint-free bandwidth. Table I summarizes the optimized dimensions of the five studied designs.

\section{A. Single SIW LWA $(N=1)$}

For the single SIW LWA, the dimensions $W_{1}, P_{1}$ and $W_{S}$, can be directly obtained as explained in [26] to achieve the desired pointing angle at the operating frequency $\left(\theta_{G O A L}=30^{\circ}\right.$ at 15 $\mathrm{GHz}$ ). The values are summarized in Table $\mathrm{I}$ for $\mathrm{N}=1$. With these dimensions, Fig. 4 shows the goal reflection-phase $\Psi_{G O A L}(f)$ for a squint-free response in the frequency band from $12 \mathrm{GHz}$ to $18 \mathrm{GHz}$. Also, the magnitude $\left|\rho_{R}(f)\right|$ and the phase $\Psi_{R}(f)$ of the reflection coefficient $\rho_{R}$ for this $\mathrm{N}=1$ design are plotted. As shown in Fig. 4, the reflection-phase condition $\Psi_{R}(f)=\Psi_{G O A L}(f)$ for leaky-mode radiation at fixed $\theta_{R}=30^{\circ}$ is only satisfied at the design frequency of $15 \mathrm{GHz}$. The goal reflection phase $\Psi_{G O A L}(f)$ has a positive slope with frequency, which cannot be followed by the reflection phase $\Psi_{R}(f)$ of a single row of PRS posts, which has a negative phase gradient as shown in Fig. 4.

The frequency response for the directivity of this $\mathrm{N}=1 \mathrm{SIW}$ LWA design has been simulated using HFSS software for three different finite antennas of lengths $L=16 \mathrm{~cm}\left(L=8 \lambda_{0}\right.$ at $\left.15 \mathrm{GHz}\right)$, $L=20 \mathrm{~cm}$ and $L=32 \mathrm{~cm}$, all of them scanning at the desired angle $\theta=\theta_{G O A L}=30^{\circ}$ at $15 \mathrm{GHz}$. Fig. 5 represents the normalized 
TABLE I

OPTIMIZED DIMENSIONS OF COUPLED SIW LWA FOR $\theta_{G O A L}=30^{\circ}$

\begin{tabular}{|l|l|l|}
\hline $\mathrm{N}$ & SIW Width $W_{i}(\mathrm{~mm})$ & Vias Periodicity $P_{i}(\mathrm{~mm})$ \\
\hline 1 & $W_{l}=7.45 W_{S}=1.8$ & $P_{l}=3.6$ \\
\hline $2 \mathrm{a}$ & $W_{l}=7.37 W_{2}=8.27 W_{S}=2.52$ & $P_{l}=3.76 P_{2}=7.75$ \\
\hline $2 \mathrm{~b}$ & $W_{l}=7.29 W_{2}=7.98 W_{S}=2.58$ & $P_{l}=3.91 P_{2}=7.75$ \\
\hline $2 \mathrm{c}$ & $W_{l}=7.23 W_{2}=7.88 W_{S}=2.58$ & $P_{1}=4.06 P_{2}=7.75$ \\
\hline 3 & $W_{l}=7.19 W_{2}=6.93 W_{3}=6.9 W_{S}=2.99$ & $P_{l}=3.57 P_{2}=3.29 P_{3}=3.37$ \\
\hline
\end{tabular}

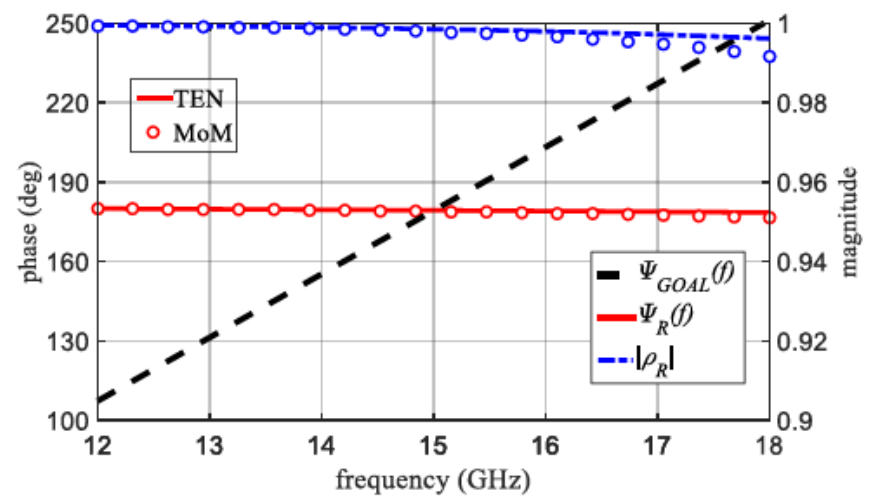

Fig. 4. Reflection coefficient for single-cavity SIW LWA with dimensions given in Table I with $\mathrm{N}=1$, and reflection-phase goal function for $\theta_{\mathrm{GOAI}}=30$.

directivity frequency response obtained in the frequency range from $14 \mathrm{GHz}$ to $16 \mathrm{GHz}$ for the three antenna lengths. Due to the inherent frequency-scanning behavior of LWAs [1], a beam is scanned from near-broadside $\left(\theta=0^{\circ}\right)$ at low frequencies to endfire $\left(\theta=90^{\circ}\right)$ direction as frequency is increased. As a result, the frequency response of the directivity at the desired angle $\theta=\theta_{G O A L}=30^{\circ}$ shows a maximum at the design frequency of 15 $\mathrm{GHz}$. The directivity at $30^{\circ}$ drops above and below this design frequency of $15 \mathrm{GHz}$, resulting in a limited $3 \mathrm{~dB}$ pattern bandwidth (PBW). Longer LWAs provide narrower beams (3) and higher directivities and thus reduced PBW, while shorter antennas result in wider beams and higher bandwidth, as shown in Fig. 5. In any case, this is the common behavior of LWAs, which limits its performance for broadband point-to-point applications as commented in the introduction of this paper.

\section{B. Two Coupled SIW LWA (N=2)}

Broadband operation with a fixed angle can be obtained if the antenna reflection phase response $\Psi_{R}(f)$ provides a positive phase gradient which matches $\Psi_{G O A L}(f)$ in the desired bandwidth. Similar positive phase-gradient responses have been applied for wideband FPA radiating at broadside [19][25], [29]-[32]. In our case, this type of response can be obtained by inserting an extra row of vias with period $P_{2}$ which couples energy to an extra SIW of width $W_{2}$, thus leading to a coupled SIW LWA of order $\mathrm{N}=2$ as represented in Fig. $1 \mathrm{~b}$.

This is illustrated in Fig. 6, where the reflection coefficients (magnitude and phase) for three different coupled SIW LWA designs with $\mathrm{N}=2$ are plotted. The coupled SIW dimensions were optimized to minimize the phase-error function (14) as explained in Section II, and they are summarized in Table I. As

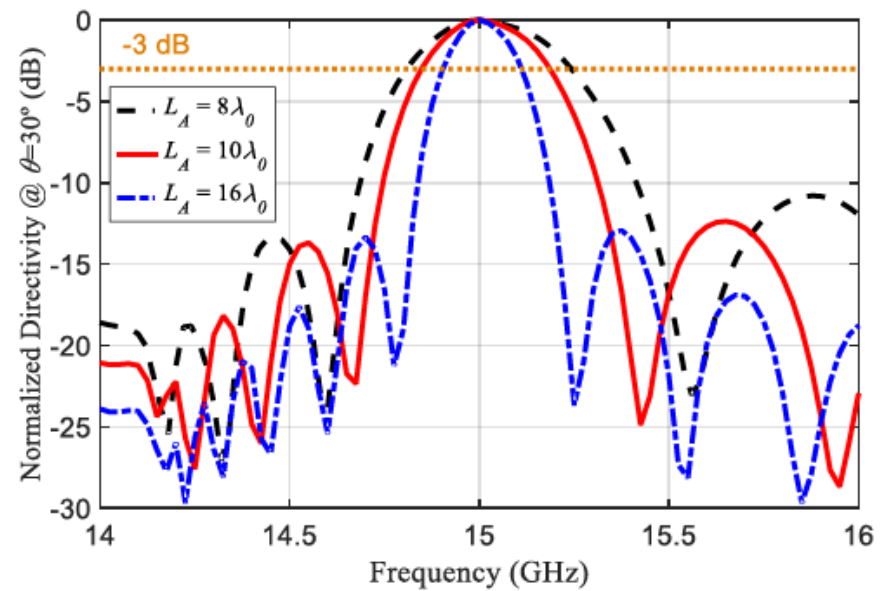

Fig. 5. Normalized directivity at $\theta=\theta_{G O A I}=30$ as a function of frequency of the $\mathrm{N}=1$ SIW LWA with dimensions given in Table I for three different antenna lengths.

can be observed, the reflection phase $\Psi_{R}(f)$ now presents a positive slope which satisfies the squint-free condition $\Psi_{R}(f)=\Psi_{G O A L}(f)$ for $\theta_{G O A L}=30^{\circ}$ not only at $15 \mathrm{GHz}$, but in given bandwidth starting at $15 \mathrm{GHz}$ and ending at $15.31 \mathrm{GHz}$ for case $\mathrm{N}=2 \mathrm{a}$ (Fig. 6a), $15.52 \mathrm{GHz}$ for case $\mathrm{N}=2 \mathrm{~b}$ (Fig. 6b), and 15.65 $\mathrm{GHz}$ for case $\mathrm{N}=2 \mathrm{c}$ (Fig. $6 \mathrm{c}$ ). Also, the reflection magnitude $\left|\rho_{R}(f)\right|$ is different to the $\mathrm{N}=1$ case, showing a resonant behavior around the design frequency of $15 \mathrm{GHz}$.

To better compare the three different designs, the resulting phase-error functions $\Delta \Psi(f)(14)$ are plotted in detail in Fig. 7. It can be observed that the squint-free condition $\Delta \Psi(f)=0$ is satisfied at $15 \mathrm{GHz}$, and also at two extra frequency points above, which can be distributed closer or further apart to determine the resulting free-squint bandwidth. The case $\mathrm{N}=2 \mathrm{a}$ in Fig. 7 shows a squint-free range from $15 \mathrm{GHz}$ to $15.31 \mathrm{GHz}$, the case $\mathrm{N}=2 \mathrm{~b}$ from $15 \mathrm{GHz}$ to $15.52 \mathrm{GHz}$, and the case $\mathrm{N}=2 \mathrm{c}$ from $15 \mathrm{GHz}$ to $15.65 \mathrm{GHz}$.

Although one could think that more separated squint-free frequency points provide a wider free-squint bandwidth, this is not strictly true. Actually, the condition $\theta_{R}=\theta_{G O A L}=30^{\circ}$ is guaranteed only at the $2 \mathrm{~N}-1$ frequency points where the TEN reflection-phase error function $\Delta \Psi(f)$ is zero. As shown in Fig. 7 , intermediate frequency points provide higher phase error as the frequency zeros are more separated (see $\mathrm{N}=2 \mathrm{c}$ in Fig. 7). On the contrary, closer zeroes provide a flatter response of the error function (see $\mathrm{N}=2 \mathrm{a}$ in Fig. 7). As the error function $\Delta \Psi(f)$ becomes higher, the condition $\theta_{R}=\theta_{G O A L}=30^{\circ}$ is less certain and it is expected the associated leaky-mode pointing angle $\theta_{R}$ to be more distant from the desired angle of $30^{\circ}$.

To obtain the variation with frequency of the leaky-mode pointing angle $\theta_{R}(f)$ with respect to the desired fixed angle $\theta_{G O A L}$, a leaky-mode dispersion analysis must be carried out. An approximate dispersion analysis can be performed by inserting the complex reflection coefficient $\rho_{R}(f)$ obtained for the optimized designs (shown in Fig. 6) in (11). Then, the TRE (11) is solved for the unknown complex transverse propagation constant $k_{x}(f)$, from which the leaky-mode longitudinal propagation constant $k_{y}(f)$ can be derived using (8). Finally, 


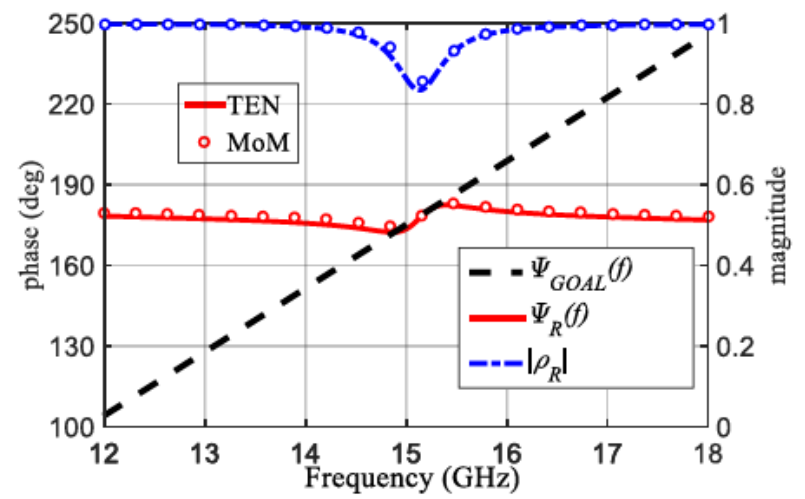

a)

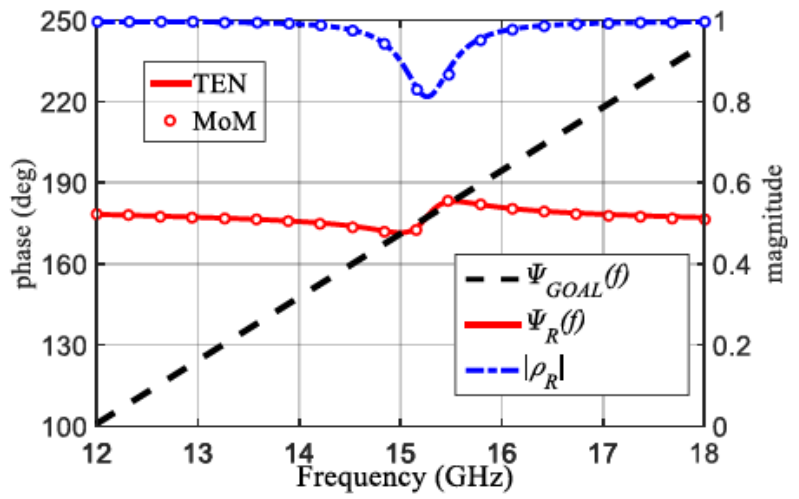

b)

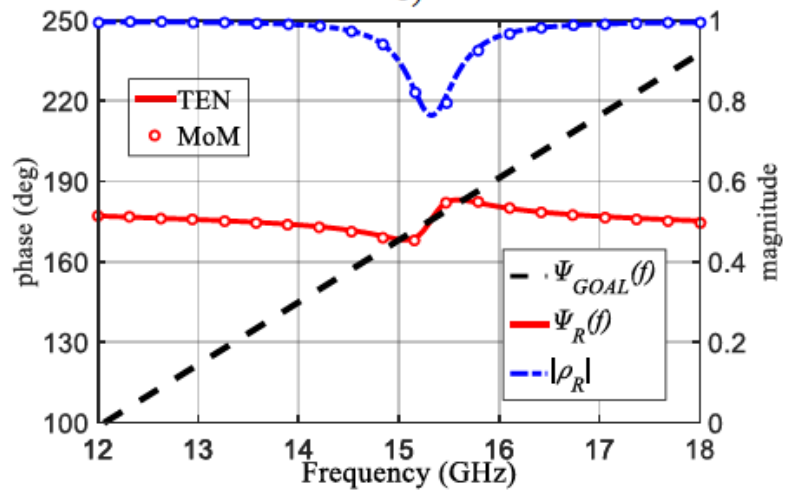

c)

Fig. 6. Reflection coefficient for optimized coupled SIW LWA with $\mathrm{N}=2$ and dimensions given in Table $I$, and reflection-phase goal function for $\theta_{\text {GOAL }}=30$.

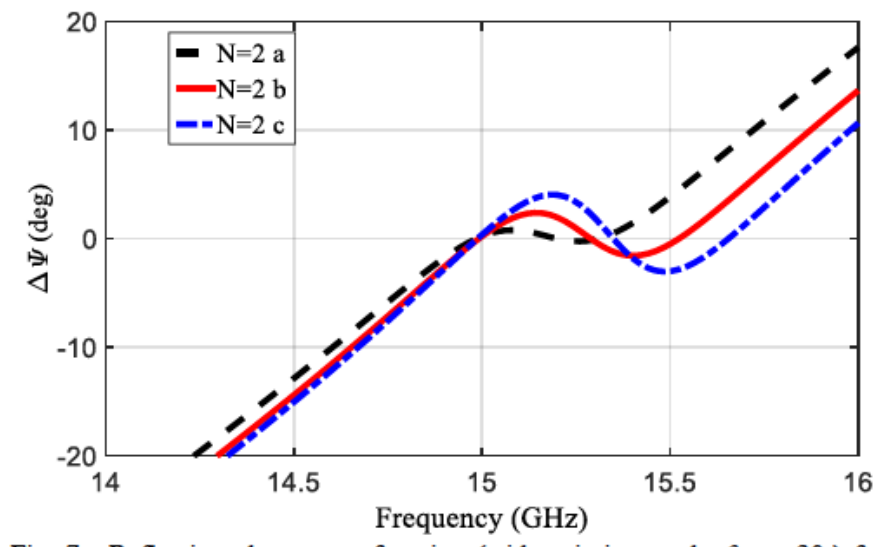

Fig. 7. Reflection-phase error function (with pointing angle $\theta_{\text {GOAL }}=30$ ) for optimized coupled SIW LWA designs of Table I with $\mathrm{N}=2$. from the phase constant $\beta_{y}(f)$, the dispersion of the angle $\theta_{R}(f)$ is obtained using (2). Fig. 8 shows the frequency dispersion for $\theta_{R}(f)$ for the three SIW LWAs designs of order $\mathrm{N}=2$.

As expected, the three designs satisfy the condition $\theta_{R}=\theta_{G O A L}=30^{\circ}$ at $15 \mathrm{GHz}$ and at two more frequency points which correspond with the frequencies of zero phase-error in Fig. 7. For intermediate frequencies, the leaky-mode pointing angle fluctuates from the goal angle of $30^{\circ}$, and this deviation is more pronounced for case $\mathrm{N}=2 \mathrm{c}\left(30^{\circ} \pm 2.8^{\circ}\right)$, and less pronounced for case $\mathrm{N}=2 \mathrm{a}\left(30^{\circ} \pm 1.5^{\circ}\right)$.

The above characteristics indicate design trade-offs should be applied. In particular, since the objective is to keep the LWA pointing angle to the desired angle $\theta_{R}=\theta_{G O A L}=30^{\circ}$ and over the wider band, the trade-off relates to the location of the frequency zeros of $\Delta \Psi(f)$. Ultimately this reflects to the stability of the gain/directivity at the desired beam pointing angle $\left(30^{\circ}\right.$ in our case). For the three designs of order $\mathrm{N}=2$ (all of them with the same antenna length as for the $\mathrm{N}=1$ design, i.e. $L=16 \mathrm{~cm}$ ), the directivity at $\theta=30^{\circ}$ as obtained using HFSS is plotted in Fig. 9.

For the case $\mathrm{N}=2 \mathrm{a}$, a $3 \mathrm{~dB}$ PBW from $14.75 \mathrm{GHz}$ to 15.53 $\mathrm{GHz}(780 \mathrm{MHz})$ is obtained, showing slight improvement when compared to the $\mathrm{N}=1$ case with $430 \mathrm{MHz}$ of $\mathrm{PBW}$ (see Fig. 5). This can be attributed to the close location of the squint-free frequency points in Fig. 8. On the contrary, the case $\mathrm{N}=2 \mathrm{c}$ showed too separated frequency zeros and too strong variation in the leaky-mode pointing angle (see Fig. 8), which results in a strong drop of directivity for intermediate frequency points as observed in Fig. 9 around $15.25 \mathrm{GHz}$. Therefore, the $\mathrm{N}=2 \mathrm{~b}$ design is the one that provides the most stable directivity response, offering a PBW of $970 \mathrm{MHz}$ from $14.75 \mathrm{GHz}$ to 15.72 GHz. A trade-off between PBW and ripple can be noticed; if lower ripple is needed, the maximum bandwidth must be reduced by locating the associated zeros to closer frequencies.

As previously commented, this numerical optimization is computationally affordable thanks to the use of closed-form expressions for all the components of the TEN. A gradientbased optimization scheme has been used to minimize the error function (12), taking 1500 simulations for convergence. To validate the results, the reflection coefficient $\rho_{R}(f)$ obtained with MoM is also represented with circles in Fig. 6, showing good agreement.

\section{Three Coupled SIW LWA (N=3)}

Following a similar optimization procedure as for the $\mathrm{N}=2$ SIW LWA, a third-order $(\mathrm{N}=3)$ SIW LWA has been designed using the TEN shown in Fig. 2d. The optimized dimensions are summarized in Table I, and the resulting reflection coefficient. is plotted in Fig. 10. Again, good matching is observed between TEN and full-wave MoM results for this $\mathrm{N}=3$ design.

Fig. 11 compares the phase-error function of this $\mathrm{N}=3$ design, with the error functions obtained for $\mathrm{N}=1$ and $\mathrm{N}=2 \mathrm{~b}$. As expected, the squint-free condition $\Delta \Psi(f)=0$ is satisfied at $2 \mathrm{~N}$ $1=5$ frequency points which can be distributed in a wider bandwidth (from $15 \mathrm{GHz}$ to $15.85 \mathrm{GHz}$ ) than for the $\mathrm{N}=1$ and $\mathrm{N}=2$ cases. 


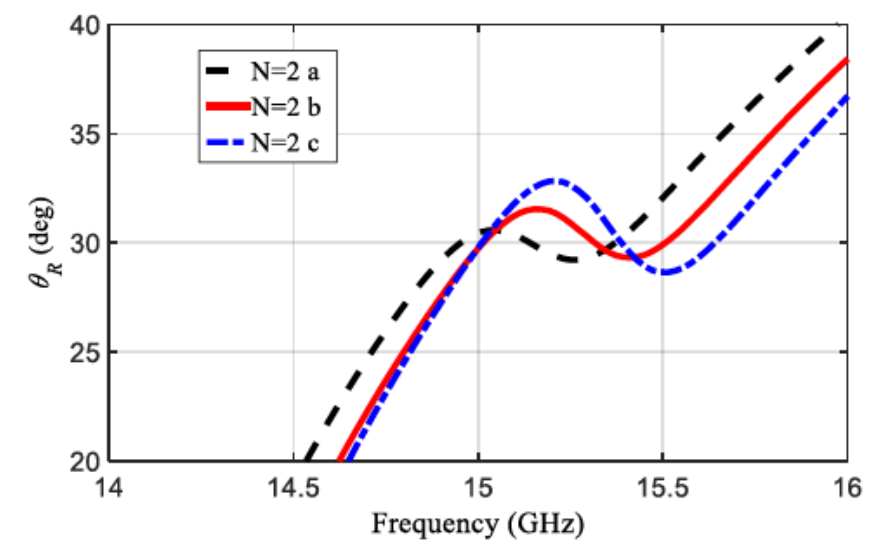

Fig 8. Dispersion of the leaky-mode angle for optimized coupled SIW LWA designs of Table I with $\mathrm{N}=2$.

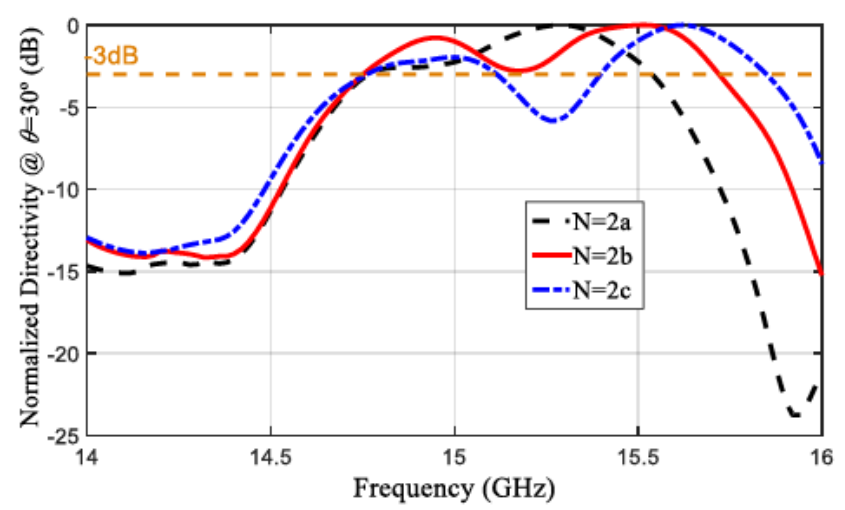

Fig. 9. Normalized directivity at $\theta=\theta_{\text {GOAI }}=30$ as a function of frequency for optimized coupled SIW LWA designs of Table I with $\mathrm{N}=2$.

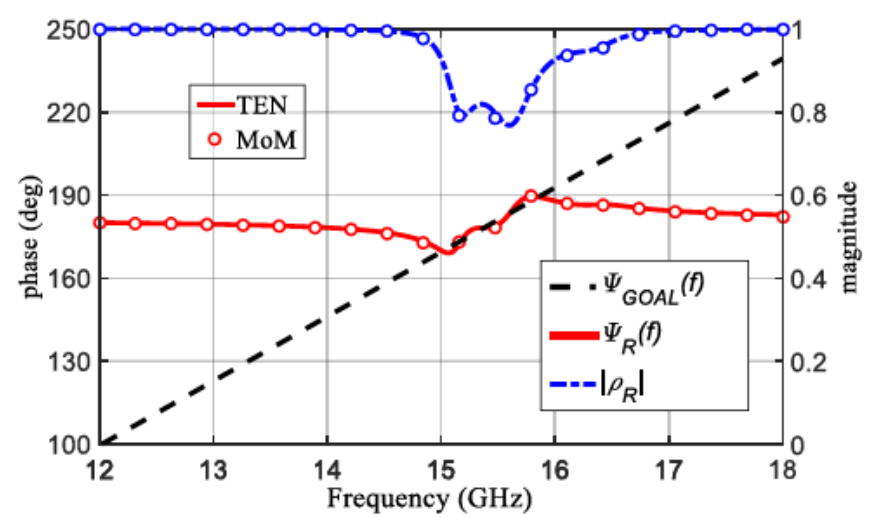

Fig. 10. Reflection coefficient for optimized coupled SIW LWA with $\mathrm{N}=3$ and dimensions given in Table $I$, and reflection-phase goal function for $\theta_{\text {GOAL }}=30$.

Fig. 12 compares the leaky-mode angle dispersion $\theta_{R}(f)$. The single SIW LWA $(\mathrm{N}=1)$ shows a conventional leaky-mode dispersion [1], [5], with a pointing angle which monotonously rises with frequency. Therefore, the condition $\theta_{R}=\theta_{G O A L}=30^{\circ} \mathrm{can}$ only be satisfied at one frequency point $(15 \mathrm{GHz})$. However, the coupled SIW designs (with $\mathrm{N}>1$ ) present a frequency band with anomalous quasi-oscillatory stabilization of $\theta_{R}(f)$ around the desired pointing angle. This type of oscillatory anomalous leaky-mode dispersion was reported in [33] for a multilayer broadband FPA radiating at broadside; this is the first time it is demonstrated for a LWA pointing off-broadside.

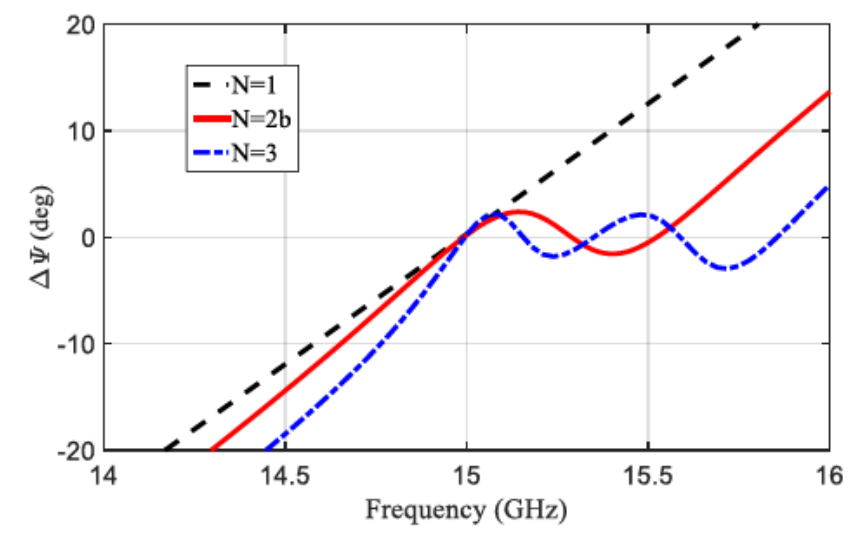

Fig. 11. Reflection-phase error function (with a goal pointing angle $\theta_{\text {GOAL }}=30$ ) for optimized coupled SIW LWA designs of Table I with $N=1, N=2 b$ and $N=3$.

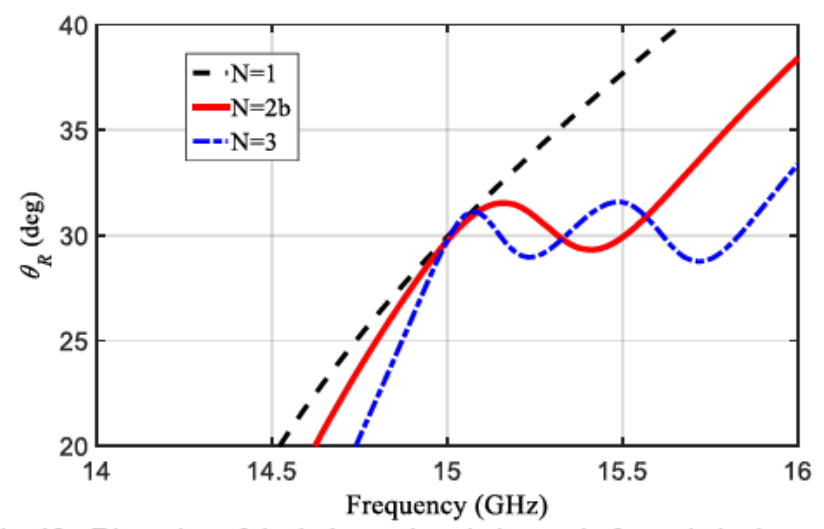

Fig. 12. Dispersion of the leaky-mode pointing angle for optimized coupled SIW LWA designs of Table I with $\mathrm{N}=1, \mathrm{~N}=2 \mathrm{~b}$ and $\mathrm{N}=3$.

In our case, the leaky-mode pointing angle $\theta_{R}(f)$ oscillates around $\theta_{G O A L}=30^{\circ}$, as shown in Fig. 12. As for the $\mathrm{N}=2 \mathrm{~b}$ case, the ripples of $\theta_{R}(f)$ have been kept within a level of $30^{\circ} \pm 1.5^{\circ}$, so that the PBW is optimized as explained in subsection III.B. The case $\mathrm{N}=3$ presents 5 frequency points which satisfy $\theta_{R}=\theta_{G O A L}=30^{\circ}$, and are distributed in the aforementioned squintfree bandwidth from $15 \mathrm{GHz}$ to $15.85 \mathrm{GHz}(850 \mathrm{MHz})$. This is an improvement compared to the $\mathrm{N}=2 \mathrm{~b}$ design that shows a squint-free bandwidth from $15 \mathrm{GHz}$ to $15.52 \mathrm{GHz}(520 \mathrm{MHz})$.

Finally, Fig. 13 shows the normalized gain at $30^{\circ}$ obtained using HFSS, for the three optimized designs with $\mathrm{N}=1, \mathrm{~N}=2 \mathrm{~b}$ and $\mathrm{N}=3$. These full-wave results demonstrate how the gain at $30^{\circ}$ presents a bandpass frequency response which half-power gain bandwidth increases with the number of coupled SIWs. Particularly, the low frequency limit in Fig. 13 is close to 14.5 $\mathrm{GHz}$, which corresponds to the onset of the leaky mode in the coupled SIW structure for the three designs. Then, the gain rises and oscillates with low ripple (below $3 \mathrm{~dB}$ ), and above a given upper frequency it drops. This behavior is expected according to the leaky-mode dispersion curves in Fig. 12. Table II summarizes the half-power gain frequency band and the resulting PBW for each design. It is obtained an increase of the bandwidth as the order of the coupled SIW LWA increases; from $2.6 \%(400 \mathrm{MHz})$ for the $\mathrm{N}=1$ antenna, to $6.4 \%(970 \mathrm{MHz})$ for the $\mathrm{N}=2 \mathrm{~b}$ antenna, and finally to $9.5 \%(1420 \mathrm{MHz})$ for the $\mathrm{N}=3$ SIW LWA design. 


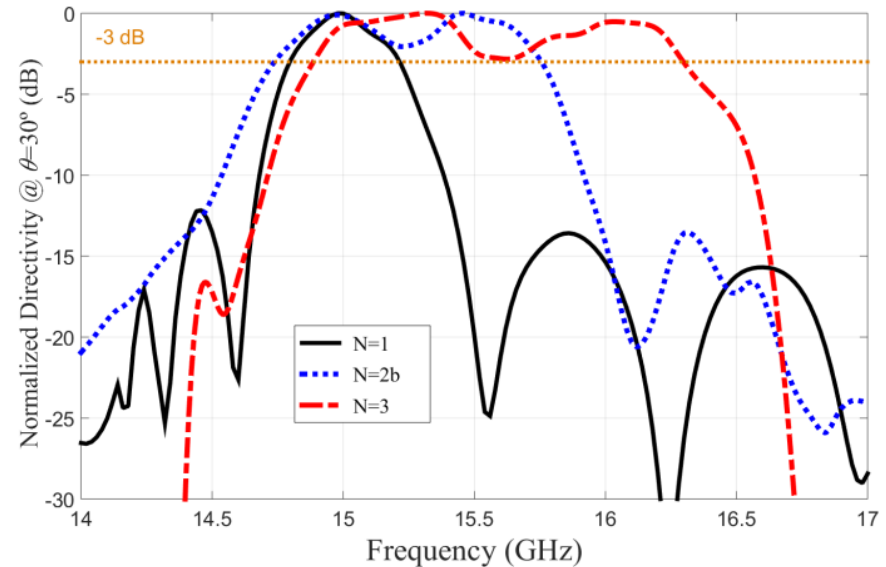

Fig. 13. Normalized gain at $\theta=\theta_{G O A L}=30$ as a function of frequency for optimized coupled-SIW LWA designs of Table I with $\mathrm{N}=1, \mathrm{~N}=2 \mathrm{~b}$ and $\mathrm{N}=3$.

TABLE II

HALF-POWER GAIN BANDWIDTH FOR DESIGNS OF TABLE I

\begin{tabular}{|c|c|c|c|}
\hline Case & $\begin{array}{c}\text { Half-power gain bandwidth } \\
\text { at } \theta=30\end{array}$ & $\begin{array}{c}\text { PBW at } \\
\theta=30\end{array}$ & $\begin{array}{c}\text { Fractional } \\
\text { BW }\end{array}$ \\
\hline $\mathrm{N}=1$ & $14.81 \mathrm{GHz}-15.24 \mathrm{GHz}$ & $400 \mathrm{MHz}$ & $2.6 \%$ \\
\hline $\mathrm{N}=2 \mathrm{~b}$ & $14.75 \mathrm{GHz}-15.72 \mathrm{GHz}$ & $970 \mathrm{MHz}$ & $6.4 \%$ \\
\hline $\mathrm{N}=3$ & $14.88 \mathrm{GHz}-16.30 \mathrm{GHz}$ & $1420 \mathrm{MHz}$ & $9.5 \%$ \\
\hline
\end{tabular}

Theoretically, it is possible to synthetize more zeros in the phase-error function (14) by increasing the order of the SIW LWA, and thus cover a wider frequency band where the squintfree condition is satisfied. However, as seen in the design with two coupled SIWs, a full-wave simulation is needed to see if the the ripple is lower than $3 \mathrm{~dB}$ and the PWB has been effectively increased. Otherwise, the dimensions of the antenna must be re-optimized and, with a higher number of coupled SIWs, the process is more complicated. Therefore, we cannot establish a theoretical limitation in the order $\mathrm{N}$ of coupled SIW, or in the effective increase in the associated gain bandwidth, but this has to be demonstrated for each optimized design. In this paper, we have demonstrated this for $\mathrm{N}=1$ to $\mathrm{N}=3$.

\section{EXPERIMENTAL VALIDATION}

This section presents experimental results with manufactured prototypes of the designed antennas, which are shown in Fig. 14. All prototypes were manufactured using commercial RTDuroid 5880 substrate $\left(H=0.508 \mathrm{~mm}, \varepsilon_{r}=2.2, \tan \delta=0.0009\right)$. As common in this type of SIW LWAs [4], [16] and also in general SIW circuits [33], [34], a width-tapered microstrip to SIW transition is used for improved matching [35], [36]. Both the microstrip and the closed (non-leaky) SIW widths are linearly tapered, so that the mode conversion and matching is performed in two stages: first from the TEM mode of the $50 \Omega$ microstrip to the $\mathrm{TE}_{10}$ guided mode of the closed (non-leaky) SIW, and then from this guided $\mathrm{TE}_{10}$ mode to the quasi-TE 10 leaky mode of the perturbed (leaky) SIW section. It is noted that matching this class of SIW LWAs is more complicated compared to other SIW components as filters [34]-[37], since SIW LWAs operate close to the cutoff frequency of the SIW mode in order to achieve leakage. As a result, the width of the radiating leaky-

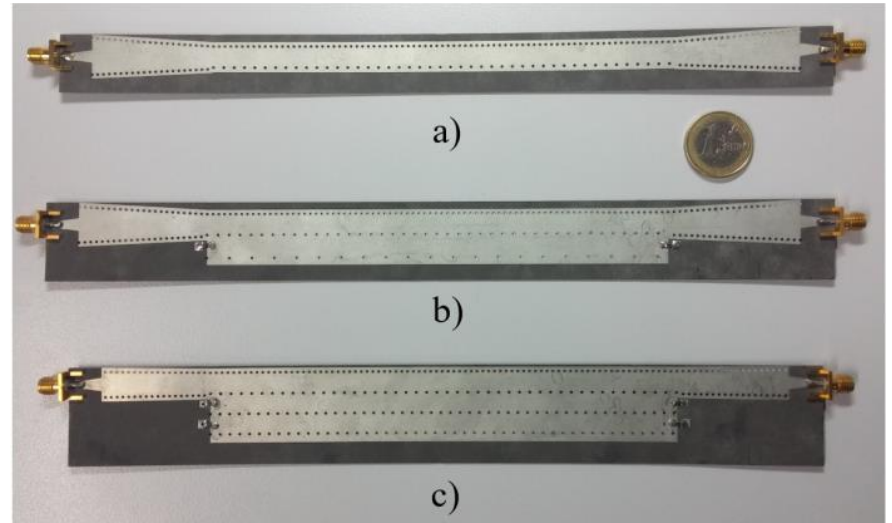

Fig. 14. Photograph of manufactured prototypes a) $\mathrm{N}=1$ b) $\mathrm{N}=2 \mathrm{~b}$ c) $\mathrm{N}=3$.

SIW section is very narrow, and a width-tapered SIW transition from a wider guiding input section to this narrow radiating section is requested, as reported in [4], [16]. A detail of this feeding mechanism is illustrated in Fig. 15 and the associated widths and lengths for each section are summarized in Table III.

The measured input matching for the three prototypes is plotted in Fig. 16 together with simulated HFSS results, observing good agreement. The three coupled-SIW LWA prototypes present a cutoff frequency close to $14 \mathrm{GHz}$, where the onset of the leaky-mode radiation occurs close to broadside direction $\left(\theta \approx 0^{\circ}\right)$. As commented, it is difficult to match the structure at this cutoff frequency. This is the reason why the bandwidth improvement is designed to be at frequencies higher than $15 \mathrm{GHz}$. This way, once the microstrip-to-SIW LWA transition for one antenna is optimized, the transition for the rest will be very similar (because they will have almost the same cutoff frequency) and easier to optimize starting from the parameters of the first design. Then, as frequency increases the matching improves showing a wide band performance with reduced $\mathrm{S}_{11}$. The impedance-matching bandwidth with $\mathrm{S}_{11}<-$ $10 \mathrm{~dB}$ covers the range from $14.7 \mathrm{GHz}$ to $17 \mathrm{GHz}$ for the three designs. As it can be seen in Fig.16, the matching at lower frequencies is degraded with adding coupled SIWs. This is due to the reduced width presented by the input SIWs for higherorder designs. As shown in Table I, $W_{l}$ is reduced from $7.45 \mathrm{~mm}$ for $\mathrm{N}=1$ to $7.29 \mathrm{~mm}$ for $\mathrm{N}=2 \mathrm{~b}$ and to $7.19 \mathrm{~mm}$ for $\mathrm{N}=3$. As a result, the input SIW presents higher cutoff frequency for higher-order designs, thus increasing the mismatch for lower frequencies.

However, the practical bandwidth of the antenna is limited by the aforementioned pattern bandwidth (PBW) for the desired pointing angle (in our case $\theta=\theta_{G O A L}=30^{\circ}$ ). The simulated and measured frequency response of the gain at the designed angle of $\theta_{G O A L}=30^{\circ}$ for the three prototypes is reported in Fig. 17 .

Good agreement is observed in Fig. 17 between the simulated and experimental results, demonstrating higher $\mathrm{PBW}$ as the order of coupled SIWs is increased. Finally, Fig. 18 compares the measured normalized gain frequency responses for the three manufactured prototypes, at the designed direction of $\theta=30^{\circ}$. Table IV summarizes the measured performance with taking into account the two main limiting factors in the overall SIW 


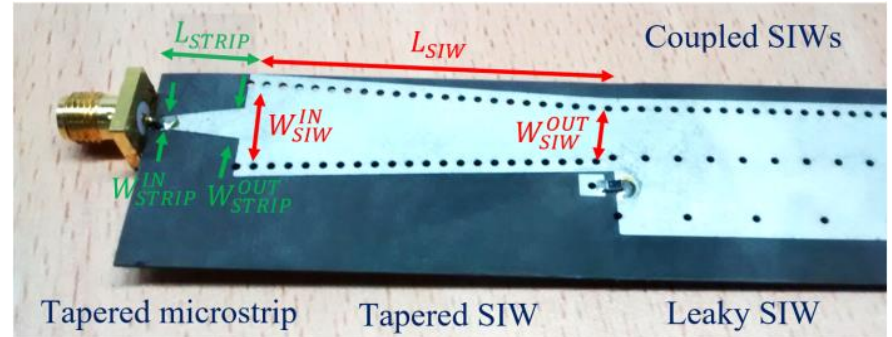

Fig. 15. Detail of microstrip-to-coupled-SIW leaky-line feeding.

TABLE III

MICROSTRIP-TO-LEAKY-SIW TRANSITION

\begin{tabular}{|c|c|c|c|}
\hline Parameter & Dimensions & Parameter & Dimensions \\
\hline$W_{\text {STRIP }}^{I N}$ & $1.62 \mathrm{~mm}$ & $W_{S I W}^{I N}$ & $12.40 \mathrm{~mm}$ \\
\hline$W_{\text {STRIP }}^{\text {OUT }}$ & $4.53 \mathrm{~mm}$ & $W_{S I W}^{\text {OUT }}$ & $7.40 \mathrm{~mm}$ \\
\hline$L_{\text {STRIP }}$ & $10 \mathrm{~mm}$ & $L_{S I W}$ & $42 \mathrm{~mm}$ \\
\hline
\end{tabular}
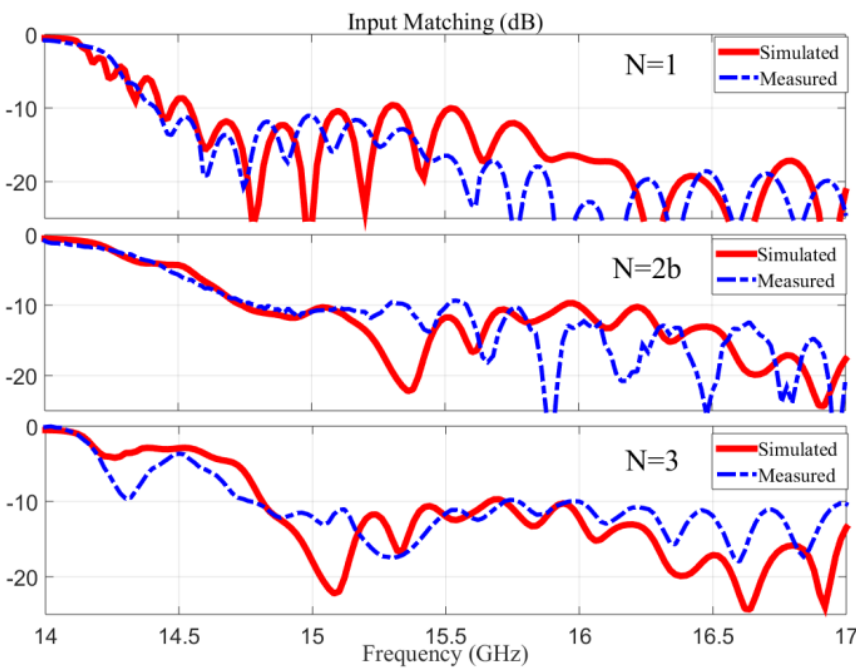

Fig. 16. Simulated and measured input matching frequency response for the three prototypes $\mathrm{N}=1, \mathrm{~N}=2 \mathrm{~b}$ and $\mathrm{N}=3$.

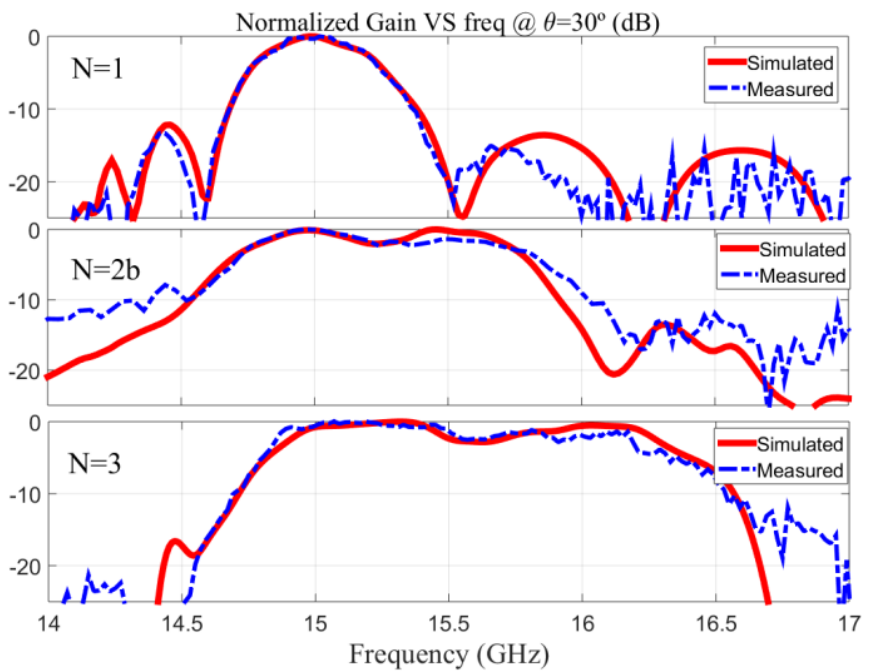

Fig. 17. Simulated and measured gain frequency response at the design pointing angle $\theta_{G O A L}=30$, for the three prototypes a) $\mathrm{N}=1 \mathrm{~b}$ ) $\mathrm{N}=2 \mathrm{~b}$ c) $\mathrm{N}=3$.

antennas bandwidth: the input impedance bandwidth and the pattern bandwidth for the pointing angle of $\theta=30^{\circ}$.

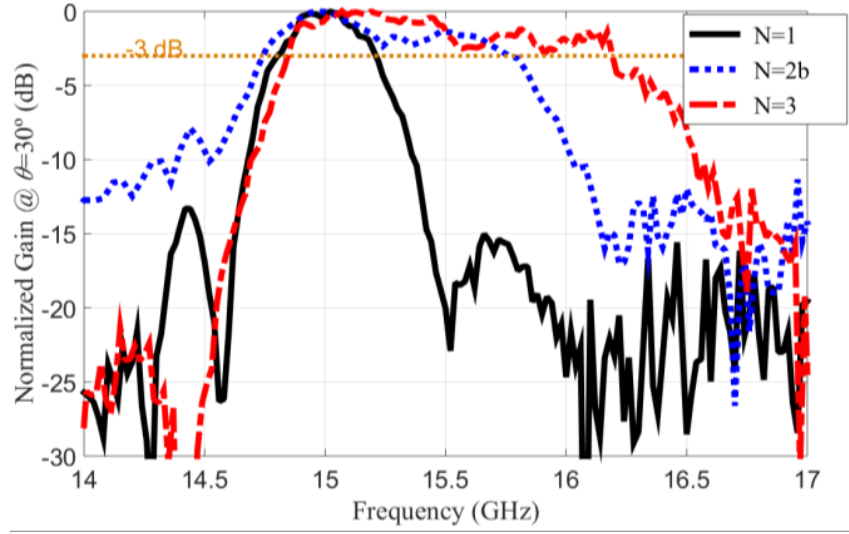

Fig. 18. Measured normalized gain vs. frequency response at angle $\theta_{G O A L}=30$, for the three prototypes a) $\mathrm{N}=1 \mathrm{~b}$ ) $\mathrm{N}=2 \mathrm{~b}$ c) $\mathrm{N}=3$.

TABLE IV

\begin{tabular}{|c|c|c|c|c|}
\hline Case & $\begin{array}{c}\text { Impedance } \\
\text { Bandwidth } \\
(\mathrm{GHz}) \\
(\mathrm{S} 11<- \\
10 \mathrm{~dB})\end{array}$ & $\begin{array}{c}\text { half-power gain } \\
\text { bandwidth at } \\
\theta=30(\mathrm{GHz})\end{array}$ & $\begin{array}{c}\text { Peak } \\
\text { Directivity } \\
\quad(\mathrm{dBi})\end{array}$ & $\begin{array}{l}\text { Peak } \\
\text { Gain } \\
(\mathrm{dBi})\end{array}$ \\
\hline $\mathrm{N}=1$ & $14.42-17$ & $14.82-15.20$ & 13.9 & 12.8 \\
\hline $\mathrm{N}=2 \mathrm{~b}$ & $14.79-17$ & $14.76-15.76$ & 12.5 & 11.5 \\
\hline $\mathrm{N}=3$ & $14.78-17$ & $14.84-16.20$ & 12.3 & 11.2 \\
\hline
\end{tabular}

The typical single-SIW LWA prototype shows a narrow gain bandwidth of $380 \mathrm{MHz}$ at $15 \mathrm{GHz}$ (i.e. 2.5\% fractional PBW), due to the well-known frequency beam squint associated to common SIW LWAs. The peak gain of this antenna is $12.8 \mathrm{dBi}$, produced at a frequency of $15 \mathrm{GHz}$, with an aperture length of $L=16 \mathrm{~cm}=8 \lambda_{0}$ at $15 \mathrm{GHz}$, with a directivity of $13.9 \mathrm{dBi}$ and a radiation efficiency of $77.6 \%$. The proposed new design with $\mathrm{N}=3$ coupled SIWs demonstrate a superior performance, with a PBW of $1360 \mathrm{MHz}$ and a peak gain of $11.2 \mathrm{dBi}$, produced at 15.2 GHz (and similar radiation efficiency of $77.7 \%$ for a lower directivity of $12.3 \mathrm{dBi}$ ). As usually happens, there is a tradeoff between the gain and the bandwidth. In our case this is translated into a $1.6 \mathrm{~dB}$ drop in gain and directivity, for an improvement of 3.6 times of the associated pattern bandwidth (from $380 \mathrm{MHz}$ to $1360 \mathrm{MHz}$, i.e. from 2.5\% to 9\% fractional bandwidth).

The directivity reduction for higher order $N$, can be attributed to lower aperture efficiency created by the interaction of multiple coupled leaky modes, which can radiate at different angles. This can be observed in the measured radiation patterns shown in Fig. 19. Effectively, while $\mathrm{N}=1$ order LWA design shows the typical single scanned beam pattern, higher order designs produce several peaks in the H-plane radiation patterns, which might be distributed at different scanning angles (see for instance the evident formation of two peaks in the $\mathrm{N}=2 \mathrm{~b}$ design at $15.36 \mathrm{GHz}$ in Fig. 19c). Obviously, this reduces the associated antenna directivity. However, it is indeed the contribution of different leaky modes to radiation at the desired angle $\theta=30^{\circ}$ for different frequencies, what makes the resulting gain bandwidth to increase for this angle for higher order $\mathrm{N}$ designs. The radiation patterns have been plotted for three frequencies inside 


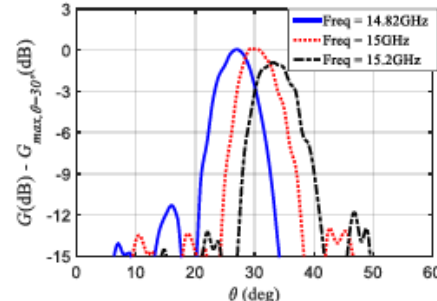

a)

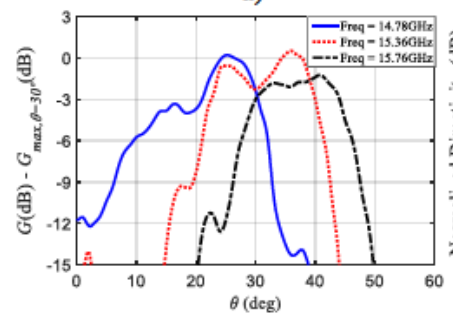

c)

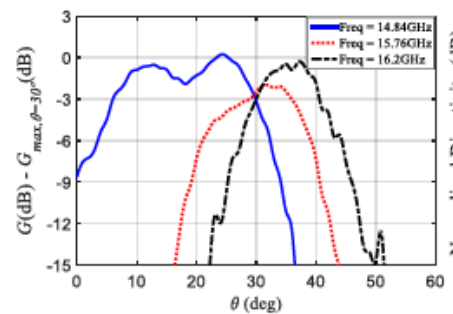

e)

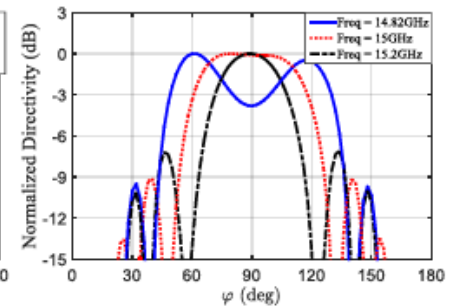

b)

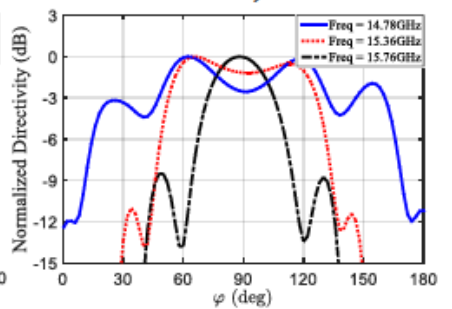

d)

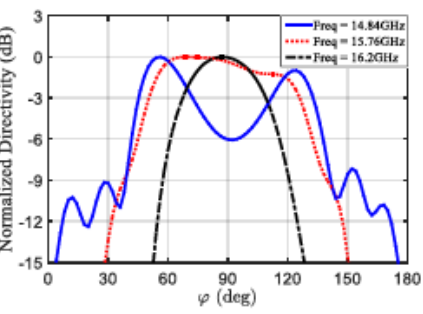

f)
Fig. 19. Measured H-plane radiation patterns $(\varphi=90)$ for different frequencies in the designed antennas bandwidth a) $\mathrm{N}=1$, c) $\mathrm{N}=2 \mathrm{~b}$, e) $\mathrm{N}=3$. Measured $\mathrm{E}$ plane radiation patterns $(\theta=30)$ b) $N=1, d) N=2 b, f) ~ N=3$.

the PBW of each design. They are normalized to the maximum gain, so that it is it is easy to observe that in the upper and lower frequencies inside the $\mathrm{PBW}$ for each design, a $3 \mathrm{~dB}$ drop in the gain occurs at the angle $\theta=30^{\circ}$. Also, the respective E-plane patterns (for $\theta=30^{\circ}$ ) are shown in Fig. 19, showing the typical fan beam which is less directive in this plane perpendicular to the antenna.

On the other hand, the variation of the radiation efficiency with frequency for the three designed antennas, is depicted in Fig. 20. It must be observed again that this radiation efficiency is understood as the relation between the directivity and the gain at the angle of interest $\theta=30^{\circ}$. This efficiency evaluates how efficiently the power is being radiated to this design angle $\theta=30^{\circ}$ at any frequency inside the antenna bandwidth. As it can be seen, the three designs present efficiency values over $60 \%$ in all the bandwidth.

\section{CONCLUSION}

It has been demonstrated that by suitably coupling leaky SIW lines, the beam-squint effect associated to directive SIW LWAs can be reduced, obtaining a wider gain bandwidth for a designed elevation angle. The proposed coupled-SIW LWA topology maintains the planar, single feeding, and passive properties of SIW LWAs. The structure is extremely integrated, thanks to the use of longitudinally coupled SIW guides along the antenna length. In this way, it is avoided the use of bulky 3D lenses, non-Foster active arrays, or more complicated nonreciprocal structures reported in previous squint-free LWAs

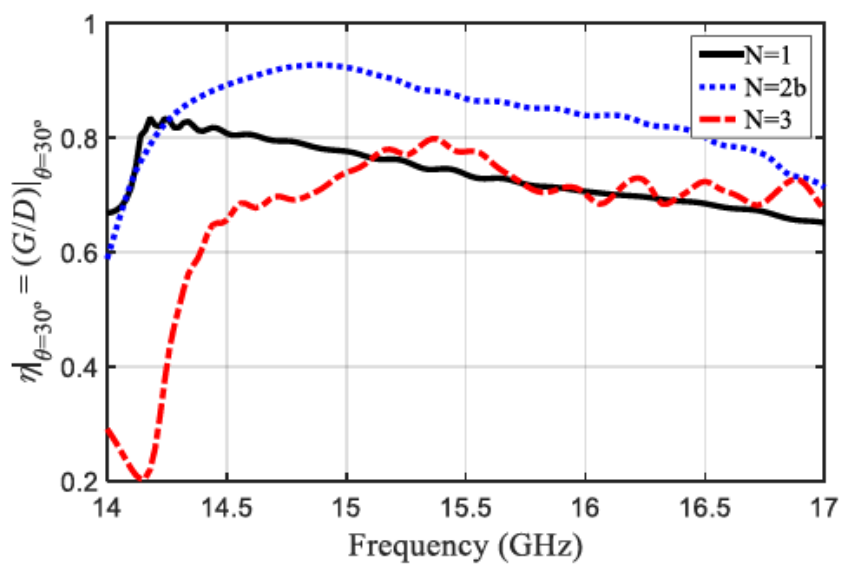

Fig. 20. Radiation efficiency for the three SIW LWA designs of order $N=1$, $\mathrm{N}=2 \mathrm{~b}$ and $\mathrm{N}=3$ at $\theta=30$ from $14 \mathrm{GHz}$ to $17 \mathrm{GHz}$.

technologies.

The TEN used to design this wideband SIW LWA, suggests that coupled-cavity filter synthesis techniques could be applied for a systematic design of this type of planar high-gain SIW antennas with application for broadband point-to-point wireless communications.

\section{REFERENCES}

[1] A. A. Oliner and D. R. Jackson, "Leaky-wave antennas," in Antenna Engineering Handbook, J. L. Volakis, Ed., 4th ed. New York: Mc- GrawHill, Jun. 2007, ch. 11.

[2] A. Sanada, H. Kubo, S.-I. Matsuzawa, and K. Sato, "Automotive radar antenna application using balanced composite right/left-handed metamaterials", in Proc. Antennas Propag. Soc. Int. Symp., pp.398-401, Jul. 2006

[3] M. Ettorre, A. Neto, G. Gerini, and S. Maci, "Leaky-wave slot array antenna fed by a dual reflector system," IEEE Trans. Antennas Propag., vol. 56, no. 10, pp. 3143-3149, Oct. 2008.

[4] Y. J. Cheng, W. Hong, K. Wu and Y. Fan, "Millimeter-wave substrate integrated waveguide long slot leaky-wave antennas and two-dimensional multibeam applications," IEEE Trans. Antennas Propag., vol. 59, no. 1, pp. 40-47, Jan. 2011.

[5] J.L. Gómez-Tornero, F.D. Quesada, A.A. Melcón, G. Goussetis, A.R. Weily, and Y. Jay Guo, "Frequency steerable two dimensional focusing using rectilinear leaky-wave lenses," IEEE Trans. Antennas Propag., vol.59, no.2, pp. 407-415, Feb. 2011.

[6] A. Neto, S. Bruni, G. Gerini, and M. Sabbadini, "The leaky lens: A broadband fixed-beam leaky-wave antenna," IEEE Trans. Antennas Propag., vol. 53 , no. 10 , pp. 3240-3246, Oct. 2005.

[7] A. Neto, "UWB, Non Dispersive Radiation From the Planarly Fed Leaky Lens Antenna- Part 1: Theory and Design," IEEE Trans. Antennas Propag., vol. 58, no. 7, pp. 2238-2247, Jul. 2010.

[8] C. Caloz, S. Abielmona, H. Nguyen, and A.Rennings, "Dual composite right/left-handed (D-CRLH) leaky-wave antenna with low beam squinting and tunable group velocity", Phys. Status Sol. B, vol. 244, no. 4, pp. 1219-1226, 2007.

[9] M. A. Antoniades, and G. V. Eleftheriades, "A CPS leaky-wave antenna with reduced beam squinting using NRI-TL metamaterials," IEEE Trans. Antennas Propag., vol. 56, no. 3, pp. 708-721, Mar. 2008.

[10] N. Nasimuddin, Z. N. Chen and X. Qing, "Substrate Integrated Metamaterial-Based Leaky-Wave Antenna With Improved Boresight Radiation Bandwidth," IEEE Trans. Antennas Propag., vol. 61, no. 7, pp. 3451-3457, July 2013.

[11] K. M. Kossifos and M. A. Antoniades, "Analysis of an off-broadside zero beam-squinting leaky-wave antenna using metamaterials," 2016 18th Mediterr. Electrotech. Conf. (MELECON), Lemesos, Cyprus, 2016, pp. $1-4$.

[12] A. Shahvarpour, A. Alvarez-Melcon, and C. Caloz, "Bandwidth enhancement and beam squint reduction of leaky modes in a uniaxially anisotropic meta-substrate," in Proc. Antennas Propag. Soc. Int. Symp., pp. 1-4, Jul. 2010. 
[13] A. Porokhnyuk, T. Ueda, Y. Kado, and T. Itoh, "Nonreciprocal metamaterial for non-squinting leaky-wave antenna with enhanced beam steering", in Proc. Antennas Propag. Soc. Int. Symp, pp. 2289 - 2290, Jul. 2013.

[14] D. F. Sievenpiper, "Superluminal waveguides based on non-foster circuits for broadband leaky-wave antennas", IEEE Antennas and Wireless Propagat. Lett., vol.10, pp.231-234, 2011.

[15] D. Muha, S. Hrabar, I. Krois, I. Bonić, A. Kiričenko, and D. Zaluški, "Design of microstrip non-foster leaky-wave antenna," in 21st Int. Conf. on Applied Electromag. and Comm (ICECom), Dubrovnik, pp. 1-3. 2013.

[16] A. J. Martinez-Ros, J. L. Gomez-Tornero, and G. Goussetis, "Planar leaky-wave antenna with flexible control of the complex propagation constant," IEEE Trans. Antennas Propag., vol. 60, no. 3, pp. 1625-1630, Mar. 2012.

[17] J.L. Gómez-Tornero, A. Martínez-Ros, A. Álvarez-Melcón, F. Mesa and F. Medina, "Substrate integrated waveguide leaky-wave antenna with reduced beam squint," 2013 Europ. Microwave Conf. (EuMC), Nuremberg, 2013, pp. 491-494.

[18] J.L. Gómez-Tornero, M. Poveda-García, R. Guzmán-Quirós, D. CañeteRebenaque, "Reducing the Beam Squint in Scanned Leaky-Wave Antennas using Coupled SIW Cavities", in Proc. Antennas Propag. Soc. Int. Symp, pp. 77 - 78, Jul. 2016.

[19] A. P. Feresidis, J. C. Vardaxoglou, "A broadband high-gain resonant cavity antenna with single feed", Proc. EuCAP 2006, Nice, France, 2006.

[20] M. A. Al-Tarifi, D. E. Anagnostou, A. K. Amert and K. W. Whites, "Bandwidth Enhancement of the Resonant Cavity Antenna by Using Two Dielectric Superstrates," IEEE Trans. Antennas Propag., vol. 61, no. 4, pp. 1898-1908, April 2013.

[21] C. Mateo-Segura, A.P. Feresidis, and G. Goussetis, "Bandwidth Enhancement of 2-D Leaky-Wave Antennas With Double-Layer Periodic Surfaces," IEEE Trans. Antennas Propag., vol.62, no.2, pp. 586-593, Feb. 2014.

[22] K. Konstantinidis, A. P. Feresidis and P. S. Hall, "Multilayer Partially Reflective Surfaces for Broadband Fabry-Perot Cavity Antennas," IEEE Trans. Antennas Propag., vol. 62, no. 7, pp. 3474-3481, July 2014.

[23] K. Konstantinidis, A. P. Feresidis and P. S. Hall, "Broadband SubWavelength Profile High-Gain Antennas Based on Multi-Layer Metasurfaces," IEEE Trans. Antennas Propag., vol. 63, no. 1, pp. 423427, Jan. 2015.

[24] B. P. Chacko, G. Augustin and T. A. Denidni, "FPC Antennas : C-band point-to-point communication systems.," in IEEE Ant. and Prop. Mag., vol. 58, no. 1, pp. 56-64, Feb. 2016.

[25] A. Chaabane, F. Djahli, H. Attia, L.M. Abdelghani, and T.A. Denidni, "Wideband and high-gain EBG resonator antenna based on dual layer PRS”, Microw. Opt. Technol. Lett., vol.59, pp. 98-101, Jan. 2017.

[26] A. J. Martinez-Ros, J. L. Gomez-Tornero, and F. Quesada-Pereira, "Efficient analysis and design of novel SIW leaky-wave antenna," IEEE Antennas Wireless Propag. Lett., vol. 12, pp. 496-499, 2013.

[27] N. Marcuvitz, Waveguide handbook. P. Peregrinus on behalf of the Institution of Electrical Engineers, Ch.5, Sec.5-21, "Inductive posts", pp. 285-289, 1986.

[28] E. Kuester, R. Johnk, and D. Chang, "The thin-substrate approximation for reflection from the end of a slab-loaded parallel-plate waveguide with application to microstrip patch antennas," IEEE Trans. Antennas Propag., vol. 30, no. 5, pp. $910-917$, Sep. 1982.

[29] G. Yuehe, K.P. Esselle, and T.S. Bird, "The use of simple thin partially reflective surfaces with positive reflection phase gradients to design wideband, low-profile EBG resonator antennas", IEEE Trans. Antennas and Propag., vol. 60, no.2, pp. 743-750, Feb. 2012.

[30] B. A. Zeb, G. Yuehe, K.P. Esselle, Z. Sun, and M. E. Tobar, "A simple dual-band electromagnetic band gap resonator antenna based on inverted reflection phase gradient", IEEE Trans. Antennas and Propag., vol. 60, no. 10 , pp. 4522-4529, Oct. 2012.

[31] N. Wang, Q. Liu, C. Wu, L. Talbi, Q. Zeng and J. Xu, "Wideband fabryperot resonator antenna with two complementary FSS layers," in IEEE Transactions on Antennas and Propagation, vol. 62, no. 5, pp. 2463-2471, May 2014.

[32] A. Hosseini, F. Capolino and D. R. Jackson, "Leaky-wave explanation of gain-bandwidth-enhanced Fabry-Pérot Cavity antennas formed by a thick multilayer partially-reflective surface," in Proc. Antennas Propag. Soc. Int. Symp., Vancouver, BC, pp. 1090-1091, July 2015.

[33] D. Deslandes and K. Wu, "Integrated microstrip and rectangular waveguide in planar form," IEEE Microwave Wireless Comp. Lett., vol. 11, Feb. 2001.
[34] D. Deslandes and K. Wu, "Single-substrate integration technique of planar circuits and waveguide filters integrated microstrip and rectangular waveguide in planar form," IEEE Trans. Microwave Theory Tech., vol. 51, pp.338-339, Feb. 2003.

[35] D. Deslandes, "Design equations for tapered microstrip-to-substrate integrated waveguide transitions," in Proc. IEEE MTT-S Intern.Microwave Symp., May 2010, pp. 1-1.

[36] E. Miralles, H. Esteban, C. Bachiller, A. Belenguer, and V. E. Boria, "Improvement for the design equations for tapered microstrip-tosubstrate integrated waveguide transitions," in Proc. Intern. Conf. Electromagnetics Adv. Appl., Sep. 2011, pp. 652-655.

[37] G. L. Matthaei, L. Young, and E. M. T. Jones, Microwave Filters, Impedance Matching Networks and Coupling Structures. New York: McGraw-Hill, 1965. 